\title{
Implantable Collamer Lens for Correction of Refractive Errors in Patients with Keratoconus following Collagen Cross-Linking: One Year Follow-up
}

\author{
Hassenien Safa Shuber
}

\begin{abstract}
Objective: The purpose of this study is to assess the predictability, safety, efficacy and stability of correction of refractive errors in patients with keratoconus using Visian implantable collamer lens (ICL) following collagen cross-linking.
\end{abstract}

Materials and methods: This prospective, non-comparative study evaluated 20 eyes of 14 patients with keratoconus subjected to implantation of intraocular collamer lens at least 1 year following collagen cross-linking and stable refraction. Mean preoperative myopia was $-8.43 \pm 5.13$ Diopters (D) $(-3.5$ to $-20.0 \mathrm{D}$ and mean cylinder was $-2.19 \pm 1.28 \mathrm{D}(0.0$ to $-4.5 \mathrm{D})$. The preoperative spherical equivalent (SE) was $-9.52 \pm 5.33 \mathrm{D}$ (-3.75 to $-21.25 \mathrm{D})$. During the 12 months follow-up, uncorrected visual acuity, best corrected visual acuity, refraction, intraocular pressure and vaulting are measured.

Results: At 12 months postoperatively, $90 \%$ of eye had refractive cylinder of $\angle 1.0 \mathrm{D}$ with $(p=0.003)$ and $20(100 \%)$ eyes was within $\pm 0.75 \mathrm{D}$ of sphere at 1 year $\left(r^{2}=0.99\right)$ and $(p=0.0085)$. The mean postoperative BCVA in decimal was $0.498 \pm 0.232$, while the mean preoperative BCVA was $0.484 \pm$ 0.285 . The safety index at 12 months was 1.02 . The mean postoperative UCVA in decimal was $0.498 \pm 0.232$ and the mean preoperative BCVA is $0.484 \pm 0.29$ and the efficacy index at 12 months was 1.01

Conclusion: Implantation of Visian Intraocular collamer lens is predictable, safe and effective way to correct refractive error in patients with keratoconus following collagen cross-linking.

Keywords: Keratoconus, Intraocular collamer lens, Collagen cross-linking.

How to cite this article: Shuber HS. Implantable Collamer Lens for Correction of Refractive Errors in Patients with Keratoconus following Collagen Cross-Linking: One Year Follow-up. Int J Kerat Ect Cor Dis 2014;3(1):29-35.

Source of support: Nil

Conflict of interest: None

\section{INTRODUCTION}

Keratoconus is the most common primary ectasia. It is usually a bilateral, asymmetric corneal degeneration characterized

Lecturer

Department of Surgery, Mustansiriya Medical College, Iraq

Corresponding Author: Hassenien Safa Shuber, Lecturer Department of Surgery, Mustansiriya Medical College, Iraq Phone: 009647902209574,e-mail: hassanienshuber@yahoo.com by localized corneal thinning which leads to protrusion of the thinned cornea. ${ }^{1}$ Corneal protrusion causes myopia and irregular astigmatism, affecting visual quality. ${ }^{2}$ It usually become apparent during the second decade of life, although the disease has been found to develop earlier and latter in life, and it typically progress until the fourth decade of life when it usually stabilizes. ${ }^{3}$ A recent study has determined that $50 \%$ of nonaffected eyes of subjects with unilateral keratoconus will develop the disease in 16 years. ${ }^{3}$

The optical management of keratoconus is by mean spectacles or contact soft contact lenses at initial stages. But, as the disease progresses, corneal distortion and irregular astigmatism become higher and the correction must be made with rigid gas permeable contact lenses to provide a regular refractive surface, thus improving visual acuity. ${ }^{4}$ In advanced stages, a penetrating keratoplasty may be necessary due to poor vision provided by contact lenses (usually worse than 6/12), the patients' intolerance to contact lenses, or, in very advanced cases, the corneal opacity due to hydrops. Also when corneal astigmatism $<10 \mathrm{D} .{ }^{4}$

Laser in situ keratomileusis (LASIK) is contraindicated in keratoconus as it may worsen the disease by further thinning the cornea. ${ }^{5}$ Intracorneal ring segments can regulate the cornea and reduce irregular astigmatism. ${ }^{6}$

The use of Phakic intraocular lenses to treat refractive errors in patients with keratoconus, have been recently described and showed effectiveness.

Clinical results of posterior phakic intraocular lenses (pIOLs) have confirmed this procedure as a promising treatment option for patients who cannot undergo keratorefractive procedures. ${ }^{8}$ The main advantages are the correction of higher levels of myopia and astigmatism and the reversibility of the procedure. ${ }^{9}$

Presently one of the most successful pIOL, known as implantable collamer lens (ICL), is the Visian ICLV4 manufactured by STAAR surgical (Monrovia, CA). ${ }^{10}$

Several studies have been published on an anterior chamber iris fixed toric pIOL, demonstrating a significant reduction in spherical and astigmatic refractive errors. ${ }^{11}$ However, concerns still exist regarding potentially induced progressive endothelial cell loss. ${ }^{12}$ The recently published outcomes of the toric ICL showed good efficacy and predictability. ${ }^{13}$ 
Corneal collagen cross-linking is a technique which aims to increase rigidity and biomechanical stability of the cornea. It involves removing the corneal epithelium in a $7 \mathrm{~mm}$ diameter central zone followed by riboflavin $0.1 \%$ solution application and corneal radiation with UV-A light at $370 \mathrm{~nm} .{ }^{14} \mathrm{UV}-\mathrm{A}$ radiation activates the riboflavin generating a reactive oxygen species that induce covalent bonds between collagen fibrils in the corneal stroma. The irradiation level at the corneal endothelium, lens and retina is significantly smaller than the damage threshold. ${ }^{15}$ It has been recommended not to perform this technique in corneas thinner than $400 \mu \mathrm{m}$ as toxic reactions could take place in the cornea. ${ }^{13}$ Several long-term studies on subjects underwent corneal cross-linking have reported an improvement in best corrected visual acuity, a flattening of keratometric readings and reduction in cone progression. ${ }^{13}$ Also, this technique has been successfully used in combination with other surgical techniques, such as corneal ring segments. ${ }^{16}$

The aim of this study is to assess the efficacy, safety, predictability and the stability of using the ICL for correction of myopia and myopic astigmatism in patients with keratoconus who have been subjected to collagen cross-linking at least 12 months before the implantation of the ICL.

\section{PATIENTS AND METHODS}

We prospectively followed 20 eyes from 14 patients with keratoconus, all of them had been subjected to collagen crosslinking at least 12 months before the implantation of ICL/ toric ICL and followed up for stability of refraction during this period. Mean patient age was 28 years (20-34 years). Mean preoperative myopia was $-8.43 \pm 5.13 \mathrm{D}$ (Diopters) ( -3.5 to $-20.0 \mathrm{D})$, and mean refractive cylinder was $-2.19 \pm$ $1.28 \mathrm{D}(0.0$ to $-4.5 \mathrm{D})$. The preoperative spherical equivalent (SE) was $-9.52 \pm 5.33 \mathrm{D}(-3.75$ to $-21.25 \mathrm{D})$. The mean anterior chamber depth was $3.3 \mathrm{~mm}$ (2.84 to 3.8$) \mathrm{mm}$. All eyes had keratometric value of $>55 \mathrm{D}$ and refractive cylinder $\leq 4.5 \mathrm{D}$.

The inclusion criteria were Best spectacle corrected visual acuity (BSCVA) of 6/37.5, age of 20 years and older, clear central cornea and sable refraction on follow-up of at least 12 months following collagen cross-linking.

Exclusion criteria were age younger than 20 years, anterior chamber depth $<2.8 \mathrm{~mm}$, cataract, glaucoma, retinal detachment, history intraocular inflammation and previous intraocular surgery. Patients with unstable refraction were also excluded from study.

Before the implantation of intraocular collamer lens (ICL), patients had complete ophthalmologic assessment and examination including the uncorrected visual acuity (UCVA), BSCVA, refraction, slit-lamp microscopy examination, Goldmann applanation tonometry, fundus exami- nation with $+90 \mathrm{D}$ biconvex lens under dilated pupil, corneal topography and pachymetry.

The diagnosis of keratoconus was established based on topographic maps acquired using Galilei Dual Scheimpflug Camera (from Ziemer Inc.) and/or topolyzer Vario (Wavelight, Alcon).

The STAAR visian intraocullar collamer lens available in spherical and in toric forms. Both forms are almost identical in term of size, shape and thickness. However, the toric form adds a cylinder element to address the patient's refractive astigmatism. ${ }^{7}$

The ICL power calculation performed through an online calculator provided by the manufacturer and can be accessed by surgeons who completed the registration process according to STAAR surgical requirements. The ICL designed to be implantation in horizontal $(0-180)$ degrees axis and can be rotated by no more than $22.5^{\circ}$ from the horizontal meridian. ${ }^{8}$ Each toric ICL provided with a rotation map in cases where rotation is needed. All surgeries in this study were performed by one surgeon. Facial block and topical anesthesia used in all cases.

Informed consent stating the benefits and the potential risks of ICL implantation was obtained from all patients prior to surgery.

In bilateral cases, the eye with higher refractive errors is operated first and the second eye is operated 7 to 10 days later.

On day of surgery, $5 \%$ phenylephrine and tropicamide $0.5 \%$ instilled for pupil dilatation 15 mintues before surgery, facial block is done by same anesthetist in all cases. A drop of tetracaine $0.1 \%$ is given every 5 minutes for 3 times to achieve further anesthesia. No, intracameral anesthetics or antibiotics given at any step of the procedure. Five minutes before the procedure, $10 \%$ povidone Iodine applied to the lids and a drop of $5 \%$ povidone Iodine administered to the eye.

The lens is loaded in the cartridge supplied by the manufacture under microscope and on a separate table. The inside of the Cartridge lubricated with a mixture of balanced salt solution (BSS) and methylcellulose 1.4\%. The lens then loaded making sure that it is edges were symmetrically tucked inside the cartridge and then lens vaulted anteriorly. A front loading forceps (Zaldivar front loading forceps), used to pull the ICL to the cartridge tip.

In case of toric ICL, a pendullar horizontal axis marker used to identify the $\left(0^{\circ}-180^{\circ}\right)$ meridian to make it as reference so that the lens can be placed in parallel to it or other wise to any other target axis. A side port of $1 \mathrm{~mm}$ is made at $11 \mathrm{o}$ 'clock in the left eye and at $5 \mathrm{o}$ 'clock in the right one. Through these ports methylcellulose $(1.4 \%)$ is injected to the anterior chamber with the aim to fill but not to over-fill it. 
A $3 \mathrm{~mm}$ clear corneal temporal three step incision is made. The tip of the cartridge is placed next to the wound while the eye is grasped with forceps and the lens slowly injected with a screw injector. While the lens is advancing to the anterior chamber, it should be closely monitored for proper unfolding to avoid flipping upside down. To ensure this a dot like marker placed on the haptics in a way that the leading haptic marker should be on the right and the rear haptic marker on the left side. The anterior chamber may be further filled with methylcellulose and an Olive tip manipulator is introduced to the anterior chamber through the side ports and the distal haptics tucked first under the iris followed by proximal haptics. Using the line marks on cornea we checked for the toric ICL alignment. If rotation was needed we used the manipulator to gently rotate the ICL. Centration and alignment of the ICL is then verified before the remaining viscoelastic is flushed by injecting saline into the anterior chamber through the main incision to fill the anterior chamber and then pushing down on the main incision.

At the completion of the procedure, two drops of tobramycin and dexamethasone mixture (Tobradex, Alcon Inc.) instilled. Postoperatively, acetazolamide $250 \mathrm{mg}$ tablet is given. Two hours later, the eye is examined with slit-lamp to assess the vault, alignment, anterior chamber formation, pupil state, and checking for excessive rise in intraocular pressure.

Follow-up visits were done at 1 day, 1 week and at 3, 6, 12 months postoperatively. On follow-up visits, UCVA, best corrected visual acuity (BCVA), slit-lamp examination, vault of the ICL away from anterior lens capsule and the intraocular pressure are all assessed. The vault is assessed in comparison to the predetermined corneal thickness in micrometers (i.e. one quarter, one half, 3 -quarters, etc.).

The same technician, who was unaware of the objectives of the study, evaluated all patients.

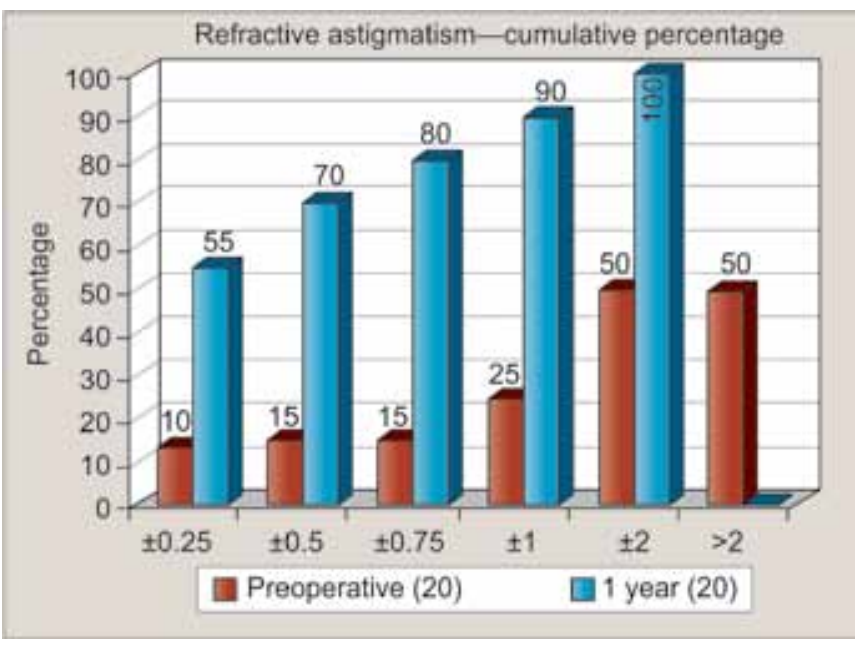

Fig. 1: Preoperative vs 12 months postoperative cylinder in diopters after ICL implantation
Data analysis was performed using datagraph — med 5.0 software for windows 2010 licenced for the author of this paper.

\section{RESULTS}

No complication happened during the surgical procedure. No eye needed removal or replacement of ICL and also no, rotation of the ICL is needed during the follow-up period. No pupil block was detected.

At 12 months postoperatively, the mean spherical equivalent error was $-0.26 \pm 0.42 \mathrm{D}(0.25$ to -1.75$)$, mean spherical error was $-0.04 \pm 0.58 \mathrm{D}(-1.75$ to -1.25$)$ and the mean cylinder was $-0.45 \pm 0.54(0.00$ to -2.0$)$.

\section{Predictability}

Figure 1 shows the manifest refractive cylinder preoperatively compared with 12 months postoperative visit. At the 12 months visit, $20(100 \%)$ eyes had $<2 \mathrm{D}$ of cylinder, compared with 10 (50\%) eyes preoperatively. Also, 18 $(90 \%)$ eyes having postoperative refractive cylinder of $\leq 1 \mathrm{D}$ compared with five $(25 \%)$ eyes preoperatively. Again, only three (15\%) eyes had refractive cylinder of $\leq 0.5 \mathrm{D}$ preoperatively while $14(70 \%)$ eyes had $\leq 0.5 \mathrm{D}$ at 12 months visit and $11(55 \%)$ eyes having $\leq 0.25 \mathrm{D}$ at the 12 months visit (see Fig. 1). The decrease in cylinder from preoperatively to 1 year postoperatively was statistically significant $(p=0.003)$.

At 12 months, the mean postoperative sphere dropped from $-8.43 \pm 5.13 \mathrm{D}(-3.5$ to -20.0$)$ preoperatively to $-0.04 \pm$ $0.58 \mathrm{D}$ and the mean SE dropped from $-9.52 \pm 5.33(-21.25$ to $-3.75)$ preoperatively to $-0.26 \pm 0.42 \mathrm{D}$. Thirteen $(65 \%)$ eyes was within $\pm 0.5 \mathrm{D}$ of attempted refractive outcome at 1 year, $r^{2}=0.99$ and $p=0.0015$. Figure 2 shows the refractive outcome in term of percentages within attempted.

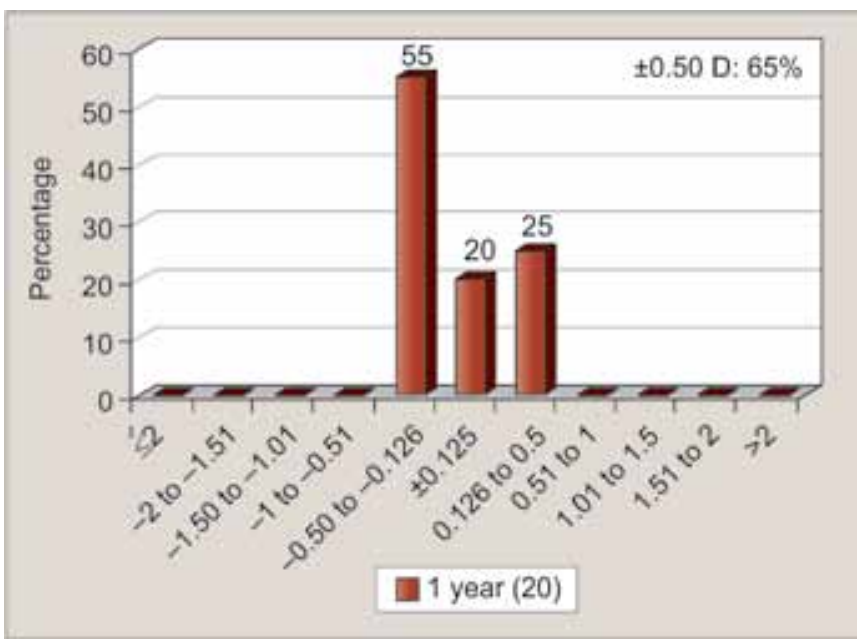

Fig. 2: Refractive outcome-percentage within attempted 
The attempted change in sphere $v s$ surgically induced refractive correction, and attempted change in astigmatism $v s$ surgically induced refractive correction are plotted in Figures $3 \mathrm{~A}$ and $\mathrm{B}$ respectively.

In Figure 3, attempted change in the sphere (3A) and attempted change in cylinder (3B) vs surgically induced refractive correction (SIRC), all in diopters (D).

The change in the astigmatic power vector preoperatively and 1 year postoperatively for all included cases shown in Figures $4 \mathrm{~A}$ and $\mathrm{B}$ respectively.

The central point of graph represents an eye with no astigmatism and the tight distribution of points around the center, 1 year postoperatively reflects the reduction of astigmatism after implantation of ICL.

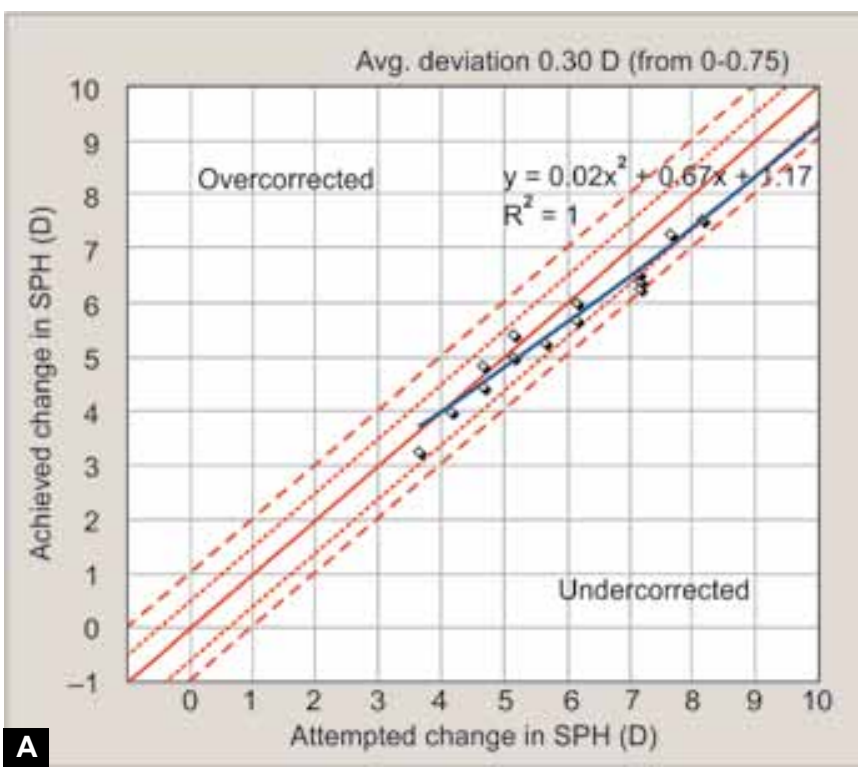

\section{Safety}

The safety results of ICL implantation shown in Figure 5. At 12 months postoperatively, none of the operated eyes had lost its BCVA, $12(60 \%)$ out of 20 studied eyes achieved their preoperative BCVA without any change, $5(25 \%)$ eyes gained two lines and another three (15\%) eyes gained $>2$ lines of BCVA. The mean postoperative BCVA in decimal was $0.498 \pm 0.232$, While the mean preoperative BCVA was $0.484 \pm 0.285$. The safety index (ratio of postoperative and preoperative BCVA) at 12 months was 1.02 (see Fig. 5).

\section{Efficacy}

Postoperatively, 15 (75\%) eyes showed CDVA of 20/40 and better, $35 \%$ showed $20 / 32$ and better, $20 \%$ showed $20 / 25$ and

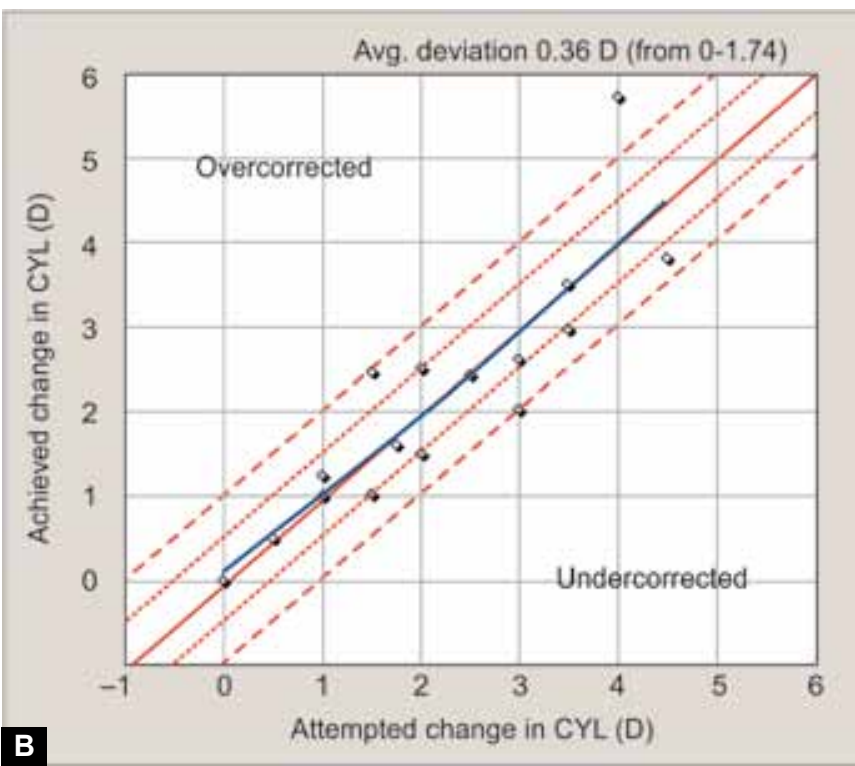

Figs 3 A and B: (A) Attempted change in sphere vs surgically induced refractive correction (SIRC) and (B) attempted change in cylinder vs surgically induced refractive correction (SIRC)-20 eyes (1 year postoperative) (Courtesy: Hassenien Safa Shuber)
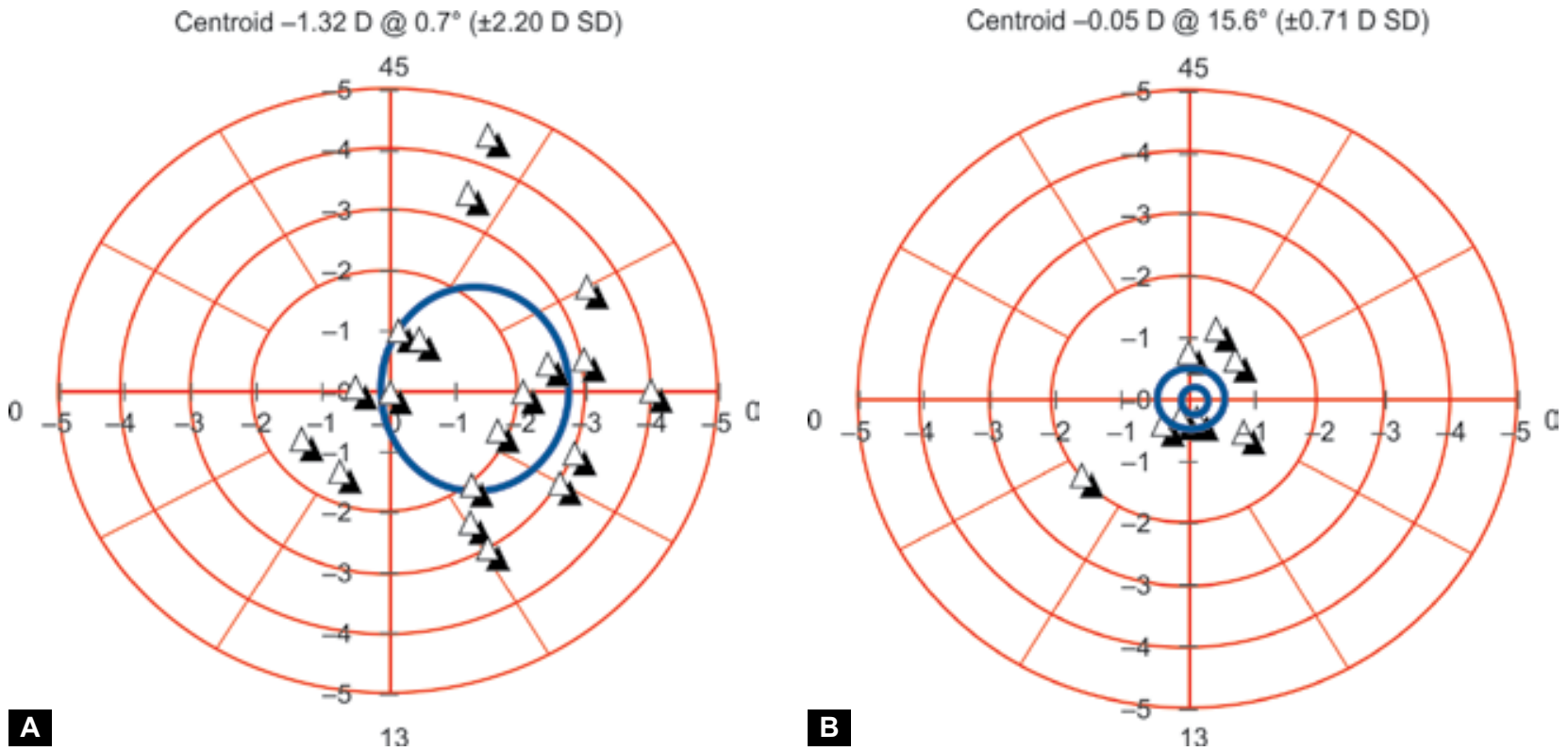

Figs 4A and B: Double angled cylinder plot for astigmatism preoperatively (A) and 1 year postoperatively (B) 
better and $15 \%$ showed $20 / 20$ and better. Figure 6 shows the cumulative CDVA preoperatively and on 12 months visit.

The preoperative UCVA was worse than $20 / 50$ in all cases, preoperative CDVA of $20 / 50$ and less seen in $60 \%$ of eyes. An increase in the number of eyes that achieved 20/40 or better from $40 \%$ ( 8 eyes) preoperatively to $75 \%$ ( 15 eyes) at 1 year postoperatively with a reduction in the number of eyes with preoperative CDVA of 20/50 and worse from $60 \%$ (12 eyes) to $25 \%$ ( 4 eyes). Figure 7 shows the preoperative CDVA vs the postoperative UCVA. The mean postoperative UCVA was $0.498 \pm 0.232$ and the mean preoperative BCVA is $0.484 \pm 0.29$ and the efficacy index (mean postoperative $\mathrm{UCVA} /$ mean preoperative BCVA) at 12 months was 1.01 .

\section{Stability}

The mean preoperative SE $-9.52 \pm 5.33 \mathrm{D}$. The mean SE was $-0.25 \pm 0.18 \mathrm{D}$ at 1 week, $-0.29 \pm 0.43$ at 1 month, $-0.27 \pm$ 0.43 at 6 months and $-0.26 \pm 0.42 \mathrm{D}$ at 1 year. The decrease in the mean SE from preoperative to 1 year postoperatively

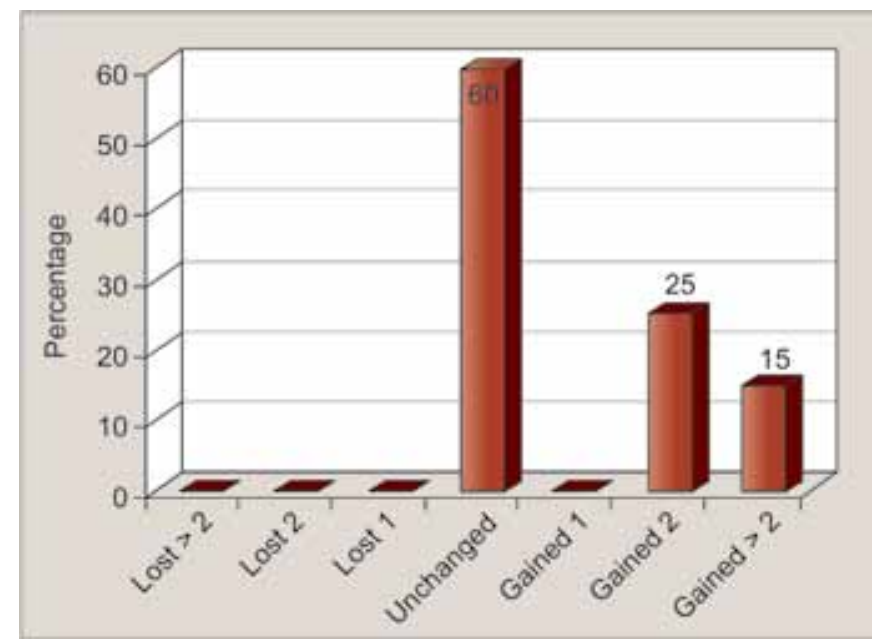

Fig. 5: Change in best corrected visual acuity after 12 months of implantation of ICL

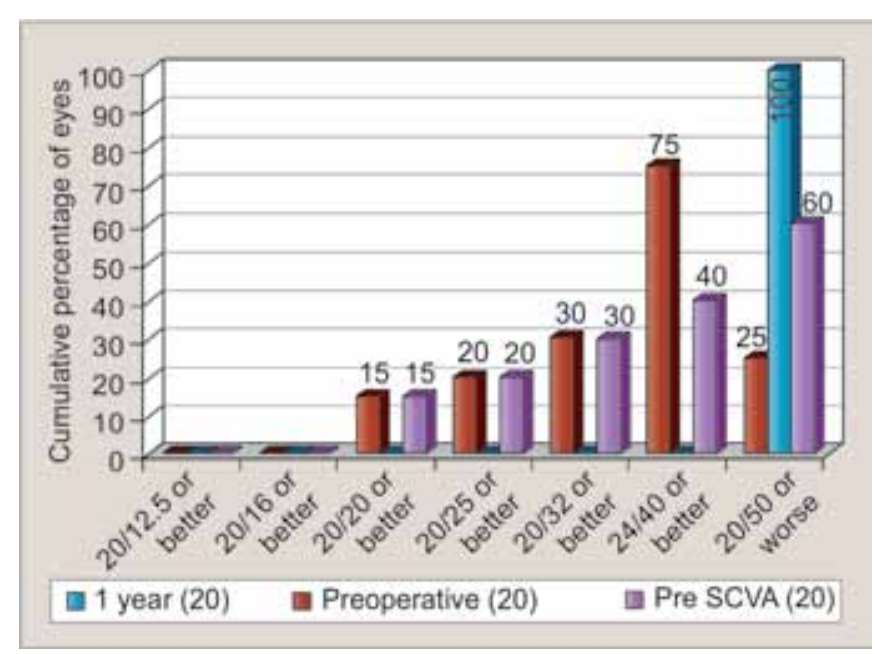

Fig. 7: Preoperative CDVA vs the postoperative UCVA-percentages was statistically significant $(\mathrm{p}<0.001)$. Figure 8 shows the improvement and stability of the mean SE over time.

Figure 9 shows the time course for refractive cylinder change from preoperative to 1 day, 1 week, 1 month, 6 months and 1 year postoperatively.

None of the examined eyes showed a change of $0.5 \mathrm{D}$ or more throughout the 1 year follow-up period. Stability data according to the FDA definition is shown in Table 1.

During the follow-up period we did not get any case of pupillary block and no, cases of chronic rise in IOP. One case of anterior subscapular cataract is seen which was visually nonsignificant after 12 months of follow. The mean IOP was $15.1 \pm 2.1 \mathrm{~mm} \mathrm{Hg}$ and the mean vault was $310 \pm$ 113 micrometers.

\section{DISCUSSION}

This prospective study of 20 eye evaluated a relatively new approach to deal with the refractive errors in keratoconic eyes. Only few studies published to address this technique.

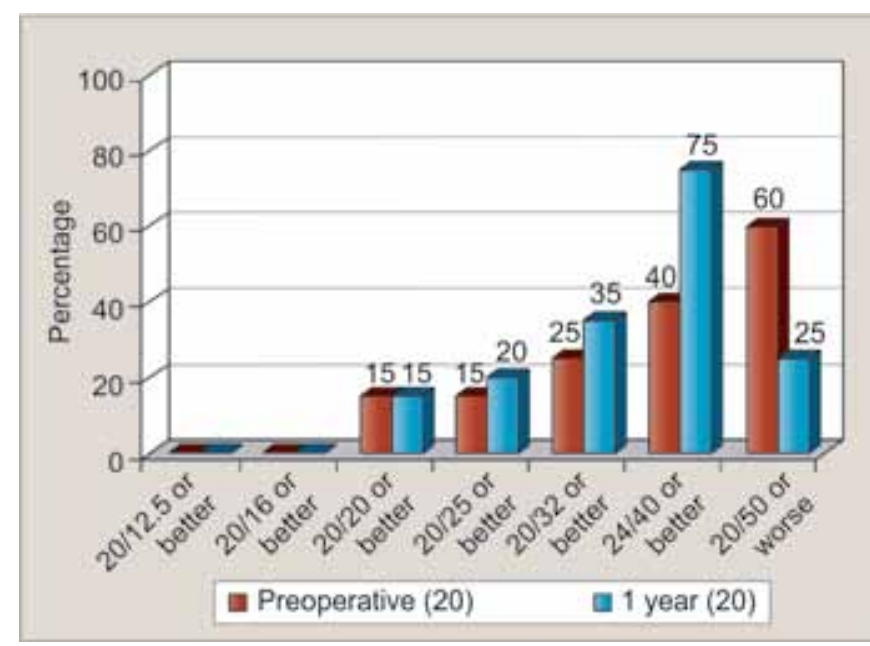

Fig. 6: The cumulative corrected distant visual acuity (CDVA), preoperatively and 1 year postoperatively

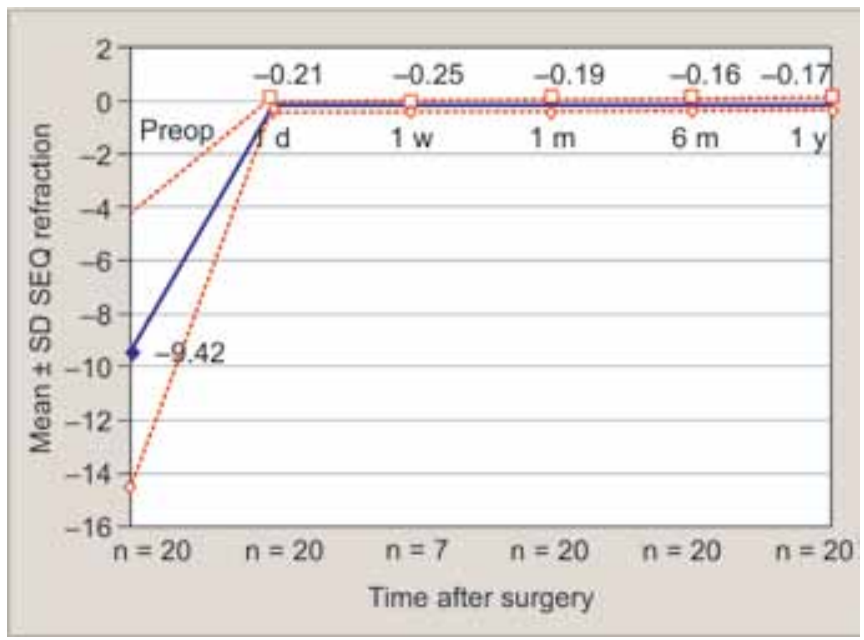

Fig. 8: The attempted vs achieved spherical equivalent (SEQ) over time 'stability'. (n: Number of eyes at given follow-up visit; SD: Standard deviation) 


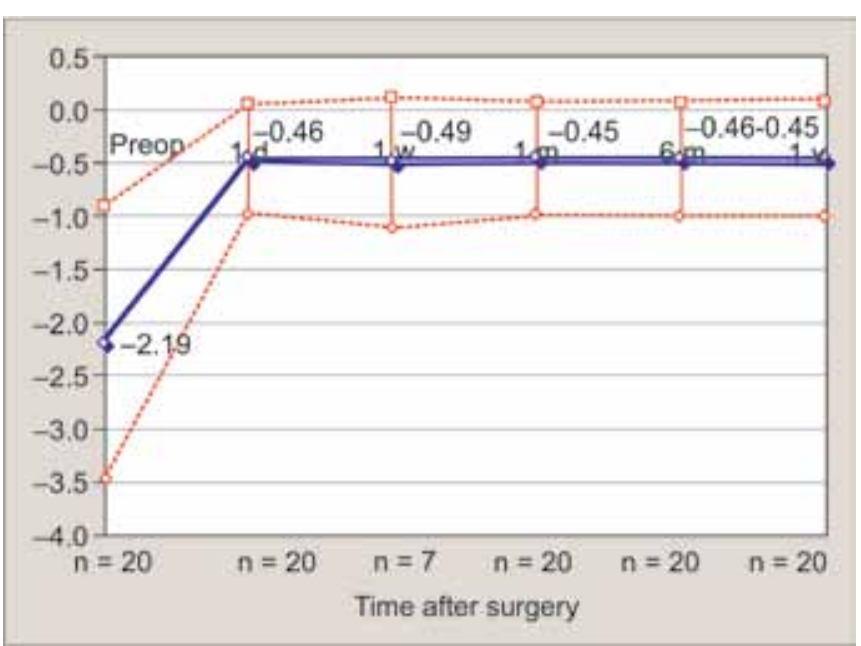

Fig. 9: Average cylinder magnitude over time ( $\mathrm{n}$ : Number of eyes at a given follow-up visit)

Our aim was to assess the efficacy, safety, predictability and stability of using ICL in these patients.

Many studies had shown that the implantation of intraocular collamer lens (ICL) is safe and effective in treating refractive errors and the united States Food and Drug Association (FDA) toric ICL 1 year clinical study was one of those studies. ${ }^{17}$ Bhikoo et al previously demonstrated that toric ICL implantation is safe, effective, predictable and with stable results over 1 year follow-up. ${ }^{18}$

Josè $\mathrm{F}$ Alfonso et al demonstrated that implantation of phakic intraocular lens (pIOL) effective, safe and stable for correction of high myopic errors after studying 138 eyes with safety index of 1.01 and efficacy index of 1.0. ${ }^{19}$

Josè $\mathrm{F}$ Alfonso et al studied the use of myopic ICL for 25 eyes from 16 patients with keratoconus and followed them for 12 months. $^{7}$

In this study, the BCVA achieved without change in 12 $(60 \%)$ eyes, 5 (25\%) eyes gained 2-lines and another $3(15 \%)$ eyes gained more than 2-lines of BCVA. None of the tested eyes lost any line of its BSCVA. The safety index was 1.02. These results can be compared to Josè F Alfonso et al study, who stated the preservation of BSCVA with no change in $72 \%$ of the test eyes, $20 \%$ gained 1 line and 2 eyes (8\%) lost 1 line of BSCVA. The safety index was 1.05 in this study.
The predictability was good. All eyes was within $\pm 2 \mathrm{D}$ of astigmatism, $18(90 \%)$ eyes was within $\pm 1.0 \mathrm{D}$ and 14 (70\%) eyes within $\pm 0.5 \mathrm{D}$. $^{7}$

Note that the astigmatism included in our study was higher than $2.19 \pm 1.28 \mathrm{D}(0.0$ to -4.5$)$ in comparison to Josè $\mathrm{F}$ Alfonso et al who had a mean cylinder of $1.24 \pm 1.19$ ( -0.5 to -3.0$)$. Josè $\mathrm{F}$ Alfonso et al in his study suggested the idea of including patients with higher astigmatic errors for such studies. ${ }^{7}$

We cannot directly compare our study to Anterior chamber pIOLs, but it may be worth mentioning that, Leccisotti and Fields ${ }^{7,20}$ reported the results found after implanting angle supported phakic IOL in 12 eyes with stage 1 and stage 2 keratoconus, mean myopia of $-10.23 \pm 2.85 \mathrm{D}(-6.5$ to -14$)$ and mean astigmatism of $-2.79 \pm 1.11 \mathrm{D}(-1$ to -5$)$. They reported that UCVA was 20/40 or better in all cases and BSCVA was equal or improved in all cases. Safety index was 1.18 and efficacy index 0.77 . The safety index seems higher but the efficacy index is less than ours (1.01). Leccisotti and Fields also said that the spherical error was corrected in all cases $\pm 1.0 \mathrm{D}$ but the astigmatism was not improved $(-2.35 \pm$ 1.0D). We get more or less similar results in term of sphere but the astigmatism improved significantly.

Leccisotti and Fields stated that the complications was only three eyes with pupil ovalization a one eye lens rotation. ${ }^{20}$ In our study, 1 eye developed anterior subcapsular cataract which was visually nonsignificant at the end of follow-up period.

Budo et al, ${ }^{21}$ presented 6 keratoconic eyes at 6 months after implantation of iris-fixated toric pIOL. Mean preoperative SE was $-18.34 \mathrm{D}$ ( -4.0 to $-29 \mathrm{D}$ ) and $-3.75 \mathrm{D}$ ( -2 to $-6 \mathrm{D})$ of astigmatism. They reported a significant reduction in SE (mean: -0.29$)$ and astigmatism (mean: $-1.33 \mathrm{D}$ ). Four out of the six eyes were within $\pm 1.0 \mathrm{D}$. Safety index was 1.49 and efficacy (1.24). They suggested high predictability in correcting the sphere than in correcting the cylinder. They concluded that iris fixated lenses might offer an alternative to treat refractive errors in patients with keratoconus.

The relative merits between posterior chamber and anterior chamber $\mathrm{pIOL}$ had been previously discussed ${ }^{20,22}$ and

Table 1: Stability (FDA definition). (Delta SEQ: Change in spherical equivalent in diopters; SD: Standard deviation in diopters)

\begin{tabular}{llll}
\hline Compare to month & Delta SEQ $(D)$ & $S D+D$ & Eyes \\
\hline Preoperative & -9.25 & 5.2041 & 20 \\
1 day & -0.0375 & 0.1223 & 20 \\
1 week & -0.0393 & 0.0675 & 7 \\
1 month & -0.0188 & 0.1167 & 20 \\
6 months & 0 & 0 & 20 \\
1 year & 0.0063 & 0.0280 & 20 \\
\hline
\end{tabular}


concern still exist regarding potentially induced progressive endothelial cell loss.

Progression of keratoconus still a concern since it may alter the refractive outcome that has been obtained in the first aftermath of ICL implantation.

A stable refraction for at least 1 year is taken as a reasonable indicator for nonprogression after collagen cross linking, however stability of keratoconus cannot be guaranteed on long-term and longer follow-up may provide the answer.

We conclude that ICL implantation offer an option to treat patient with keratoconus with clear central cornea, refractive cylinder of $\leq 4.5 \mathrm{D}$, steep $\mathrm{K}<55 \mathrm{D}$ and stable refraction over at least 12 months.

Longer follow-up and larger group of cases may be of considerable value to proof stability, predictability and safety of the technique on long-term.

\section{REFERENCES}

1. Kennedy RH, Bourne WM, Dyer JA. A 48-year clinical and epidemiologic study of keratoconus. Am J Ophthalmol 1986; 101(3):267-273.

2. Zadnikk K, Batt JT, Gordon MO, Edrington TB. Study group. Biomicroscopic signs and severity in keratoconus. Cornea 1996; 15(2):139-146

3. Romer-Jimènez M, Santodomingo-Rubido J, Wolffsohn JS. Keratoconus: a review. Contact Lens and Anterior Eye 2010;33(4):157-166.

4. Chopra I, Jain AK. Between eye asymmetry in keratoconus in an Indian population. Clin Exp Optom 2005;88(3):146-152.

5. Briefly SC, Lzquierdo L Jr, Mannis MJ. Penetrating keratoplasty for keratoconus. Cornea 2000;19(3):329-332.

6. Lim L, Pesudous K, Coster DJ. Penetrating keratoplasty for keratoconus: visual outcomes and success. Ophthalmology 2000;107(6):1125-1131.

7. Alfonso JF, Palacios A, Montes-Mico R. Myopic phakic STAAR collamer posterior chamber intraocular lenses for keratoconus. J Refrac Surg 2008;24(9):867-874.

8. Lovisolo CF, Reinstein DZ. Phakic Intraocular lenses. Surv Ophthalmol 2005;50(6):549-587.
9. Sanders DR, Doney K, Poco M. United States Food and Drug Administration Clinical Trial of Implantable Collamer Lens (ICL) for moderate to high myopia, 3 years follow-up. Ophthalmology 2004;111(9):1683-1692.

10. Arne JL, Lesueur LC. Phakic posterior chamber lenses for high myopia: functional and anatomical outcomes. J Cataract Refract Surg 2000;26(3):369-374.

11. Lackner B, Pieh S, Schmidinger G, et al. Outcome after treatment of ametropia with implantable contact lens. Ophthalmology 2003;110(11):2153-2161.

12. Bloomenstein MR, Dulaney D, Barner RW, Perkins SA. Posterior chamber phakic lens for moderate myopia and hyperopia. Optometry 2002;73(7):435-446.

13. Mertens EL. Toric phakic implantable collamer lens for correction of astigmatism: one year outcomes. Clinical Ophthalmology 2011;5:369-375.

14. Auffarth GU, Wang L, Völcker HE. Keratoconus evaluation using the orbscan topography system. J Cataract Refract Surg 2000;26(2):222-228.

15. Prisanto O, Legeais, Renard G. Superior Keratoconus. Cornea 1997;16(6):693-694.

16. Rahmen W, Anwar S. An unusual case of keratoconus. J Pediatric Ophthalmol Strabismus 2006;43(6):373-375.

17. Sanders DR, Schneider D, Martin R, Brown D, Dulaney D, et al. Toric intraocular Collamer lens for moderate to high myopic astigmatism. Ophthalmology 2007;114(1):54-61.

18. Bhikoo R, Rayner S, Gray. Toric implantable collamer lenses for patients with moderate to sever myopic astigmatism: 12 months follow-up. Clin Experiment Ophthalmol 2010;38(5):467-474.

19. Alfonso JF, Lisa C, Fernandez-Vega CL. Clinical outcome after implantation of a posterior chamber collagen copolymer phakic intraocular lens with central hole for myopic correction. J Cataract Refract Surg 2013;39(6):915-921.

20. Leccisotti A, Fields SA. Angle supported phakic IOL in eyes with keratoconus and myopia. J Cataract Refract Surg 2003; 29(8):1530-1536.

21. Budo C, Bartels MC, van Rij G. Implantation of Artizan toric phakic intraocular lenses for the correction of astigmatism and spherical errors in patients with keratoconus. J Refractive Surg 2005;21(3):218-222.

22. Van der Heijde GL. Some optical aspects of implantation of an intraocular lens in myopic eye. European J Implants Refrac Surg 1989;1(5):245-248. 